\section{Eficácia e segurança da terapia com idursulfase em pacientes com mucopolissacaridose tipo II, com e sem comparação com placebo: revisão sistemática e metanálise}

\author{
Efficacy and safety of idursulfase therapy in \\ patients with mucopolysaccharidosis type II \\ with and without comparison to placebo: \\ systematic review and meta-analysis
}

\section{Eficacia y seguridad del tratamiento con idursulfasa en pacientes con mucopolisacaridosis tipo II con y sin comparación con el placebo: revisión sistemática y metaanálisis}

\begin{abstract}
Mucopolysaccharidosis type II (MPS II) is a genetic disease of broad clinical spectrum, characterized by a deficiency of the enzyme iduronate2-sulfatase. The aim of this study was to assess whether enzyme replacement therapy (ERT) with idursulfase (IDS) for MPS II is effective and safe. PubMed/MEDLINE, Embase, LILACS, and Cochrane Library were searched until November 30, 2012. Only five articles met the inclusion criteria (randomized controlled trials - RCTs, or open-label trials/prospective case series including $\geq 5$ patients and evaluating relevant outcomes). A meta-analysis was performed for forced vital capacity (FVC; absolute and \%) and for distance walked on the 6-minute walking test (6MWT). There was a statistically significant increase, but not clinically relevant, in both variables; an increased risk for development of mild infusion-related reactions and IgG antibodies to IDS were also found. The data suggest that ERT with IDS is safe and has a potential benefit for MPS II patients, but further studies are required.

Mucopolysaccharidoses; Enzyme Replacement Therapy; Health Technology Evaluation
\end{abstract}

Taciane Alegra 1

Dauana Pitano Eizerik 2

Caio César Silva de Cerqueira ${ }^{1}$

Tiago V. Pereira 3,4

Alícia Dorneles Dornelles 5

Ida Vanessa Doederlein Schwartz 1,6,7

\section{Resumo}

A mucopolissacaridose tipo II (MPS II) é uma doença genética de amplo espectro clínico, caracterizada por deficiência da enzima iduronato-2sulfatase. Revisão sistemática avaliou a eficácia e segurança da terapia de reposição enzimática (TRE) com idursulfase (IDS) na MPS II. As bases de dados PubMed/MEDLINE, Embase, LILACS e Biblioteca Cochrane foram pesquisados até 30 de novembro de 2012. Apenas cinco estudos preencheram os critérios de inclusão (ensaios clínicos randomizados - ECRs, ECRs abertos ou séries de caso prospectivas, incluindo cinco ou mais pacientes e avaliando desfechos relevantes). Metanálise foi realizada para capacidade vital forçada (CVF; valores absolutos e em \%) e para a distância percorrida no teste da caminhada dos seis minutos, com mudanças significativas em ambas as variáveis; também foi encontrado risco aumentado de reações leves relacionadas à infusão e de desenvolvimento de anticorpos IgG à IDS. Em face dos dados apresentados neste estudo, conclui-se que a TRE com IDS é segura e tem benefício potencial em MPS II, mas estudos adicionais são necessários.

Mucopolissacaridoses; Terapia de Reposição Enzimática; Avaliação de Tecnologias de Saúde 


\section{Introdução}

A mucopolissacaridose tipo II (MPS II, síndrome de Hunter, MIM \#309900) é uma doença genética que abrange ampla variação fenotípica (apresentação clínica). Essa doença é caracterizada pela atividade deficiente da enzima lisossomal iduronato-2-sulfatase (IDS; EC 3.1.6.13); em decorrência, existe acúmulo intracelular de glicosaminoglicanos (também conhecidos como GAGs, carboidratos que atuam na formação, manutenção e reparação de tecidos estruturais) e aumento da sua excreção urinária 1.

A apresentação clínica da MPS II pode ser atenuada ou grave, dependendo do comprometimento neurológico 2,3. Dado seu padrão de herança (ligado ao X recessivo), os pacientes afetados geralmente são do sexo masculino 1,4,5,6. Manifestações comuns incluem fácies típica, obstrução de vias aéreas superiores, regurgitação valvular cardíaca, doença pulmonar restritiva, hepatoesplenomegalia, hérnias, contraturas articulares, síndrome da apneia obstrutiva do sono e redução da qualidade de vida 3,5. Achados adicionais incluem: baixa estatura, macrocefalia com ou sem hidrocefalia comunicante, macroglossia, perda auditiva, síndrome do túnel do carpo e displasia esquelética 3,5. Na forma grave, as manifestações iniciam-se por volta dos dois anos de idade, progredindo para sinais/sintomas somáticos e deficiência cognitiva durante a infância; já os pacientes com a forma atenuada apresentam os primeiros sintomas na infância tardia, com progressão da doença somática mais lenta e branda, e pouca ou nenhuma deficiência cognitiva 4,5 .

Os tratamentos específicos disponíveis para a MPS II são o transplante de células-tronco hematopoiéticas (TCTH) ou a terapia de reposição enzimática (TRE) 7. A indicação do primeiro é controversa, já que, na literatura, existem poucas evidências sobre sua eficácia e trata-se de um procedimento associado à alta morbimortalidade ${ }^{3}$. Por outro lado, a TRE envolve a aplicação endovenosa periódica de enzima recombinante semelhante à enzima que se encontra deficiente. Atualmente, encontra-se disponível a TRE com idursulfase (IDS), medicamento aprovado para tratamento da MPS II pelo Food and Drug Administration (FDA, Estados Unidos) em 2006 e pelo European Medicines Agency em 2005 (EMA, Europa), além de ter sido registrado na Agência Nacional de Vigilância Sanitária (ANVISA) em 2008. A dose preconizada é de $0,5 \mathrm{mg} / \mathrm{kg}$ por semana, em infusão que deve durar de uma a três horas, a qual, inicialmente, deve ser aplicada sem prémedicação. Entretanto, até o presente momento, persistem incertezas acerca de sua eficácia clínica e segurança.

\section{Objetivos}

Avaliar se o uso da TRE com IDS em pacientes com MPS II é eficaz, seguro e capaz de proporcionar melhora clínica e da qualidade de vida dos pacientes.

\section{Métodos}

\section{Estratégia de busca e critérios de inclusão dos estudos}

As bases de dados EMBASE, PubMed/MEDLINE e LILACS foram pesquisadas para artigos publicados até 30 de novembro de 2012. De acordo com critérios pré-definidos, seriam incluídos ensaios clínicos randomizados (ECR), nos quais se comparasse a TRE ao placebo ou a outro tratamento na MPS II. Como provavelmente haveria um pequeno número de ECRs satisfazendo os critérios de inclusão, optou-se por inserir, também, estudos com outros delineamentos: ECRs abertos, EC não randomizados (quase experimento), séries de casos contemporâneas (prospectivas), desde que a amostra de pacientes fosse igual ou superior a cinco e que fosse avaliado algum desfecho relevante, definido a priori pela equipe (Tabela 1).

As estratégias de busca foram aplicadas da seguinte forma no PubMed/MEDLINE: ( $(m u$ copolysaccharidosis II) OR (mucopolysaccharidosis type II) OR (MPS II) OR (L-sulfoiduronate sulfatase deficiency) OR (Hunter syndrome) OR ("IDS protein, human")) AND ((idursulfase) OR (Elaprase) OR (enzyme replacement therapy) OR (recombinant human iduronate-2-sulfatase)); no EMBASE e LILACS realizamos a busca expandida com as palavras-chave enzyme replacement, iduronate 2 sulfatase, Hunter syndrome, mucopolysaccharidosis II.

A revisão sistemática foi elaborada de acordo com as orientações da Cochrane Collaboration ${ }^{8}$. Dois pesquisadores (T. A. e D. P. E.) realizaram as buscas de forma independente, selecionando os artigos de interesse por meio dos títulos e resumos; após reunião de consenso, elaboraram uma lista de artigos elegíveis, os quais foram lidos na íntegra por ambos os pesquisadores. As referências dos artigos foram manualmente revisadas em busca de trabalhos adicionais que não houvessem sido identificados através da estratégia de busca.

A extração dos dados também foi realizada em duplicata de modo independente por dois revisores (T. A. e D. P. E.), utilizando um formulário padronizado. Durante esse processo, dados complementares não descritos nos artigos foram 
Desfechos considerados como relevantes para avaliação da terapia de reposição enzimática com idursulfase em pacientes com mucopolissacaridose tipo II (MPS II)

\begin{tabular}{cc}
\hline Desfechos clínicos & Desfechos substitutos \\
\hline $\begin{array}{c}\text { Acuidade visual } \\
\text { Estado nutricional } \\
\text { Estatura }\end{array}$ & $\begin{array}{c}\text { Alterações cardíacas } \\
\text { Capacidade vital forçada } \\
\text { Custo-efetividade }\end{array}$ \\
Eventos adversos (qualquer um) & Distúrbio de comportamento \\
Mobilidade articular & Esplenomegalia \\
Número de infecções respiratórias de internações hospitalares & Estágio puberal \\
Número de episódios de otites & Glicosaminoglicanos urinários \\
Peso & Hepatomegalia \\
Qualidade de vida & Hidrocefalia \\
Regressão neurológica & Mielopatia \\
Retardo mental & Opacificação de córnea \\
Síndrome da apneia obstrutiva do sono & Resistência física \\
Sobrevida & Teste da caminhada de 12 minutos \\
\end{tabular}

pesquisados nas bases de registros de ensaios clínicos (http://www.clinicaltrial.gov; http://www. controlled-trials.com; Registro Cochrane de Ensaios Controlados).

\section{Avaliação da qualidade da evidência e risco de vieses}

Para se verificar o risco de vieses, a qualidade metodológica dos artigos foi avaliada por meio do Grading of Recommendations Assessment, Development and Evaluation (GRADE) Working Group System" 9,10,11. Esta ferramenta classifica a qualidade dos desfechos em alta, moderada, baixa e muito baixa 12 , utilizando os seguintes critérios: risco de vieses, inconsistência, indiretividade, imprecisão.

\section{Síntese quantitativa da evidência}

O tempo de seguimento nos artigos selecionados variou de 23 a 104 semanas. Além disso, houve sobreposição entre os participantes de diferentes estudos publicados. Como resultado, não foram realizadas estimativas sumárias para pontos temporais específicos, mas se optou pela utilização do último ponto relatado de cada estudo e/ou o ponto com maior número de participantes. Essa abordagem é justificada não apenas porque poucos estudos relataram dados em qualquer período de tratamento, mas também porque se mostrou difícil separar os participan- tes independentes de duas ou mais publicações sobrepostas.

Os estudos elegíveis não apresentaram, na sua maioria, um grupo de controle. Dessa maneira, para variáveis contínuas, o efeito do tratamento foi resumido como diferença média pré e pós, isto é, a mudança do desfecho em relação ao apresentado no início do tratamento. Para desfechos binários, usou-se a proporção dos indivíduos experimentando um evento após o tratamento.

Para varáveis contínuas, as variâncias intraestudo foram estimadas utilizando-se a distribuição $t$. A síntese quantitativa foi realizada tanto pelo modelo de efeitos fixos, quanto pelo modelo de efeitos aleatórios. representada pelos métodos de Peto e de DerSimonian-Laird. Optou-se pelo método de Peto por essa técnica ter propriedades estatísticas superiores em cenários com eventos binários raros. A heterogeneidade estatística foi avaliada pelo teste $Q$ de Cochran e quantificada pelo índice $I^{2}$. Um valor de $\mathrm{p}<0,05$ foi considerado como evidência de efeito significativo do tratamento; no entanto, para o teste Q, tipicamente com baixo poder estatístico, um nível de significância de $\mathrm{p}<0,1$ foi escolhido. Todas as análises estatísticas foram realizadas no programa Stata 11.1 (Stata Corp., College Station, Estados Unidos). 


\section{Resultados}

\section{Características dos estudos incluídos}

Inicialmente, foram identificados 374 estudos (Figura 1), mas apenas dois eram ECRs, duploscegos, comparando IDS ao placebo 13,14, enquanto três preencheram critérios como estudos não randomizados 15,16,17. Nenhum estudo comparando TRE a outro tratamento foi encontrado e não foram obtidas informações adicionais do registro de ensaios clínicos. Foi identificada uma metanálise sobre TRE em MPS II, mas os crité- rios de inclusão ali utilizados eram diferentes dos aplicados nesta pesquisa 18 .

$\mathrm{O}$ fato de os artigos encontrados descreverem de forma não detalhada a sua metodologia e resultados prejudicou a síntese quantitativa das variáveis. Assim, foi possível fazer a metanálise apenas das seguintes variáveis: alteração da porcentagem prevista da capacidade vital forçada (CVF\%), distância percorrida no teste de caminhada de seis minutos (TC 6min), risco de desenvolvimento de reações relacionadas com a infusão e risco de desenvolvimento de anticorpos de classe IgG à IDS.

\section{Figura 1}

Terapia de reposição enzimática em mucopolissacaridose tipo II. Fluxograma da revisão sistemática da literatura.

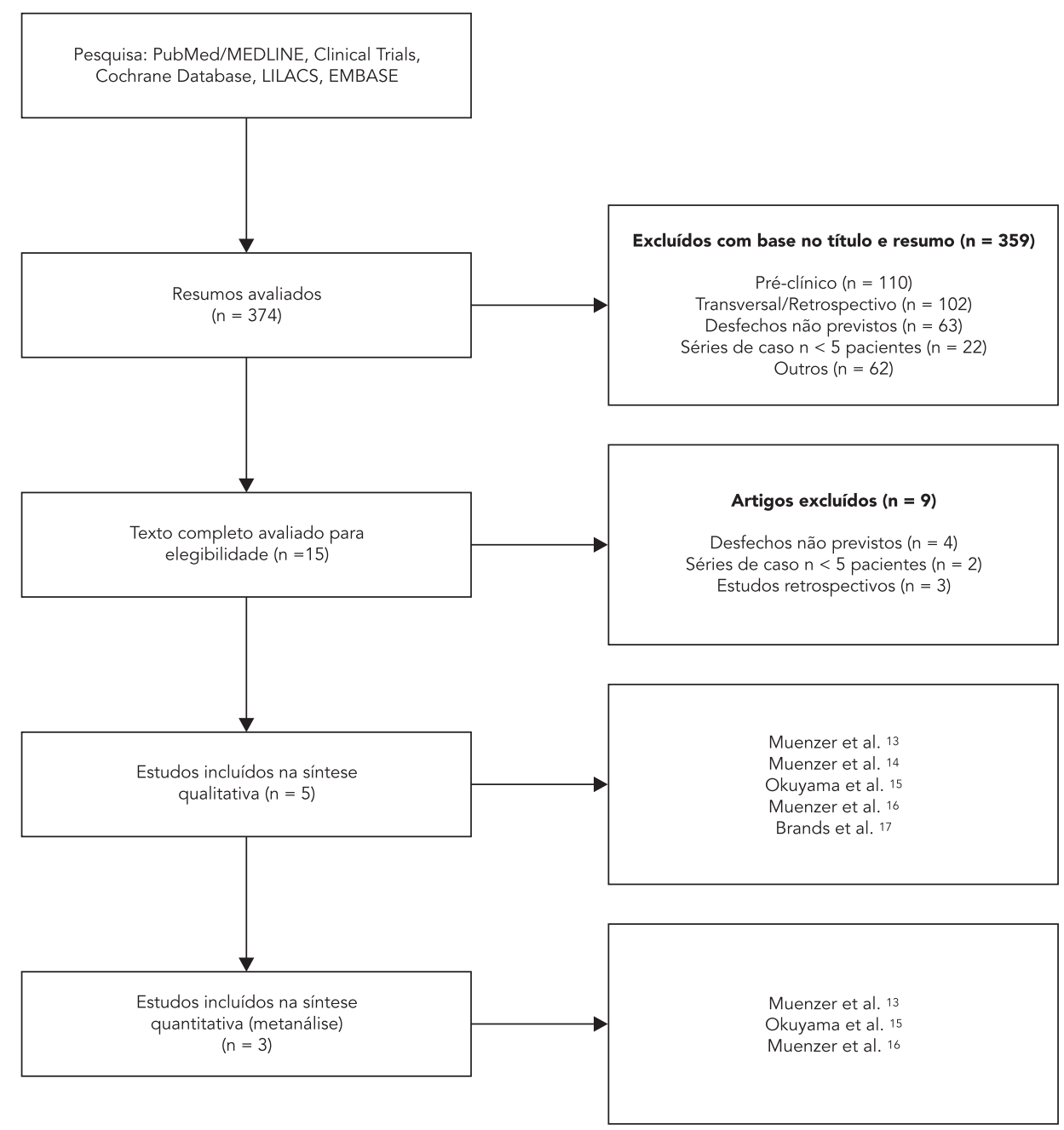


Utilizando a classificação de qualidade do GRADE, todos os desfechos foram classificados com qualidade muito baixa (em virtude, sobretudo, da indiretividade e da imprecisão), exceto para GAGs urinários e efeitos adversos, os quais foram, ambos, de qualidade moderada (apresentavam menor indiretividade em relação aos demais).

\section{Risco de vieses nos estudos incluídos}

Nos dois ECRs, o cegamento e a geração de sequência da randomização não foram adequadamente descritos 13,14. Analisando as características iniciais dos pacientes, observa-se que estes apresentavam o fenótipo atenuado da MPS II. Os ensaios abertos foram séries de casos sem grupo-controle contemporâneo para comparação. Outro problema foi a falta de relato das cointervenções aplicadas aos pacientes durante a TRE (como, por exemplo, uso de outros medicamentos ou suporte ventilatório não invasivo), as quais costumam ser indicadas em pacientes com MPS II. A comunicação incompleta e seletiva de algumas variáveis ocorreu não só nos ECRs, como também nos estudos abertos. Por exemplo, o intervalo de confiança de algumas variáveis não foi relatado e, no estudo de Muenzer et al. 14, não há avaliação estatística seja entre os valores finais e inicias para cada grupo, seja da diferença entre eles; demonstra-se apenas o cálculo dos valores pré- e pós-TRE dos três grupos combinados. Ademais, todos os estudos foram patrocinados pela indústria farmacêutica fabricante do medicamento, e dois deles apresentavam o mesmo grupo de pacientes. As observações supracitadas nos levam à conclusão de que pode existir alto risco de vieses nos trabalhos incluídos na presente revisão sistemática e metanálise.

\section{Síntese quantitativa dos dados}

Apresenta-se, na Tabela 2, uma visão geral das principais características dos estudos incluídos, com resumo dos desfechos primários definidos para cada artigo. Na Tabela 3 encontra-se um sumário de todos os desfechos de eficácia avaliados. A seguir, são descritos os resultados agrupados de acordo com a comparação: placebo ou sem grupo-controle.

\section{- Comparação 1: síntese dos efeitos da idursulfase versus placebo}

\section{a) Distância percorrida no TC $6 \mathrm{~min}$}

Esse teste consiste na medida da distância (em metros) que o indivíduo é capaz de percorrer em seis minutos. Na comparação com o placebo, não houve diferença estatisticamente significativa em um estudo 14; já em outro, a IDS $0,5 \mathrm{mg} / \mathrm{kg}$ semanal foi significativamente superior em relação ao placebo [média da mudança da distância percorrida \pm erro-padrão $(\mathrm{EP})=44,3 \pm 12,3 \mathrm{~m}$

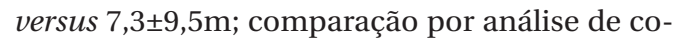
variância entre placebo e IDS ( $p=0,0013)]$, mas não no regime quinzenal $(30,3 \pm 10,3 \mathrm{~m}$ versus

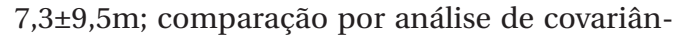
cia entre IDS e placebo $\mathrm{p}=0,073) 13$.

\section{b) Função pulmonar}

A função pulmonar foi avaliada pelo desfecho substituto CVF\% previsto. Não houve modificações estatisticamente significativas na CVF\% quando se comparou o regime quinzenal ou semanal de IDS ao placebo nos ECRs avaliados 13,14.

\section{c) Desfecho combinado: TC $6 \mathrm{~min}$ e CVF}

O estudo de Muenzer et al. 13 utilizou um escore de gravidade que combinou os resultados do TC 6min e da medida da CVF, calculado por meio do procedimento descrito por O’Brien 19: a mudança de cada parâmetro em relação ao basal era classificada independentemente do grupo de tratamento, constituindo-se como desfecho primário a soma da pontuação dos dois componentes individuais (TC e CVF\%). Houve diferença estatisticamente significativa na comparação entre placebo e IDS 0,5mg/kg semanal ( $\mathrm{p}=0,0049$ ) ou quinzenal ( $\mathrm{p}=0,042$ ), entretanto não foi constatada significância estatística se comparados os dois regimes de IDS ( $p=0,133$ ). É interessante observar que, nas análises individuais, houve diferença da IDS versus placebo apenas no TC $6 \mathrm{~min}$.

\section{d) Volume hepático e esplênico}

Em um dos estudos, há relato de redução no volume hepático após TRE com IDS na comparação com placebo, tanto no regime quinzenal, quanto no semanal (percentual de mudança $-0,8 \pm 1,6 \%$; $-24 \pm 1,7 \%$ e $-25,3 \pm 1,6 \%$, respectivamente, $\mathrm{p}<$ $0,0001)$. O mesmo foi verificado para o volume esplênico $(+7,2 \pm 4,2 \% ;-19,8 \pm 3,2 \%$ e $-25,1 \pm 2,4 \%$, respectivamente, $\mathrm{p}<0,0001)$. Para essas duas variáveis, não há relato de comparação direta entre o regime quinzenal e semanal, apenas destes versus placebo 13 .

\section{e) Mobilidade articular}

Um dos ECRs reporta os resultados de uma articulação: ganho estatisticamente significativo 
Tabela 2

Revisão sistemática sobre terapia de reposição enzimática com idursulfase na mucopolissacaridose tipo II (MPS II). Síntese dos estudos incluídos.

\begin{tabular}{|c|c|c|c|c|c|c|c|}
\hline Estudo (ano) & Delineamento & n (M:F) & $\begin{array}{c}\text { Idade mediana } \\
\text { em anos } \\
\text { (amplitude) }\end{array}$ & $\begin{array}{c}\text { Dose de IDS EV } \\
\text { (pacientes) }\end{array}$ & $\begin{array}{c}\text { Comparação } \\
\text { (pacientes) }\end{array}$ & $\begin{array}{l}\text { Duração } \\
\text { (semanas) }\end{array}$ & $\begin{array}{l}\text { Resultados } \\
\text { dos desfechos } \\
\text { primários dos } \\
\text { estudos }\end{array}$ \\
\hline $\begin{array}{l}\text { Muenzer et al. } 13 \\
(2006) *\end{array}$ & $\begin{array}{c}\text { ECR, duplo-cego } \\
\text { (Fase II/III) }\end{array}$ & $96(96: 0)$ & $14(5,4-31,0)$ & $\begin{array}{c}0,5 \mathrm{mg} / \mathrm{kg} \\
\text { semanal }(\mathrm{n}=32) \\
0,5 \mathrm{mg} / \mathrm{kg} \\
\text { quinzenal } \\
(\mathrm{n}=32)\end{array}$ & Placebo $(n=32)$ & 53 & $\begin{array}{c}\uparrow \text { na variável } \\
\text { composta CVF\% } \\
\text { e TC 6min (IDS } \\
\text { semanal vs. } \\
\text { placebo; } \\
p=0,0049 \text { ) }\end{array}$ \\
\hline $\begin{array}{l}\text { Muenzer et al. } 14 \\
\text { (2007) }\end{array}$ & $\begin{array}{c}\text { ECR, duplo-cego } \\
\text { (Fase I/II) }\end{array}$ & $12(12: 0)$ & $N R * \star(6-20)$ & $\begin{array}{c}0,15 \mathrm{mg} / \mathrm{kg} \\
\text { quinzenal }(\mathrm{n}=3) \\
0,5 \mathrm{mg} / \mathrm{kg} \\
\text { quinzenal }(\mathrm{n}=3) \\
1,5 \mathrm{mg} / \mathrm{kg} \\
\text { quinzenal }(\mathrm{n}=3)\end{array}$ & Placebo $(n=3)$ & 24 & $\begin{array}{l}\downarrow \text { GAGs urinários } \\
\quad(p=0,0007)\end{array}$ \\
\hline $\begin{array}{l}\text { Okuyama et al. } 15 \\
\text { (2010) }\end{array}$ & Estudo aberto & $10(10: 0)$ & $30(21,1-54,0)$ & $\begin{array}{c}0,5 \mathrm{mg} / \mathrm{kg} \\
\text { semanal }(n=10)\end{array}$ & Nenhum & 52 & $\begin{array}{l}\text { Não define } \\
\text { desfechos } \\
\text { primários }\end{array}$ \\
\hline $\begin{array}{l}\text { Muenzer et al. } 16 \\
(2011) *\end{array}$ & Estudo aberto & $94(94: 0)$ & $14(5,4-31,0)$ & $\begin{array}{c}0,5 \mathrm{mg} / \mathrm{kg} \\
\text { semanal }(n=94)\end{array}$ & Nenhum & 104 & $\begin{array}{c}\uparrow \text { da distância } \\
\text { no TC 6min } \\
(42 \pm 10 \mathrm{~m} ; \\
\text { p }<0,001) \\
\text { CVF (\%previsto): } \\
\text { NS }\end{array}$ \\
\hline $\begin{array}{l}\text { Brands et al. } 17 \\
\text { (2013) }\end{array}$ & $\begin{array}{l}\text { Estudo de } \\
\text { coorte }\end{array}$ & $6(6: 0)$ & $N R * \star \star(1,0-2,8)$ & $\begin{array}{c}0,5 \mathrm{mg} / \mathrm{kg} \\
\text { semanal }(\mathrm{n}=6)\end{array}$ & Nenhum & $104-159$ & $\begin{array}{c}\downarrow \text { dimensão } \\
\text { do septo } \\
\text { interventricular } \\
\text { durante a } \\
\text { diástole } \\
(-0,36 \text { escore Z/ } \\
\text { ano, p = 0,05; } \\
\mathrm{n}=5) \text { e do } \\
\text { índice de massa } \\
\text { ventricular } \\
\text { esquerda } \\
\text { (-0,26 escore Z/ } \\
\text { ano, p = 0,032; } \\
\mathrm{n}=5) ; \text { NS para } \\
\text { fração de ejeção, } \\
\text { morfologia } \\
\text { valvar ou outros } \\
\text { parâmetros } \\
\text { cardíacos }\end{array}$ \\
\hline
\end{tabular}

CVF\%: capacidade vital forçada predita; ECR: ensaio clínico randomizado; IDS EV: idursulfase endovenosa; GAGs: glicosaminoglicanos; NR: não relatado; NS: não significativo; TC 6min: teste da caminhada de seis minutos.

* Avaliado o mesmo grupo de pacientes;

** Não demonstrada mediana para o grupo completo, apenas média $=14$ anos;

*** Demonstrado apenas intervalo de idade.

Nota: Todos os estudos incluídos foram prospectivos. São mostrados somente os resultados dos desfechos primários destes estudos. 
Eficácia da terapia de reposição enzimática com idursulfase para mucopolissacaridose tipo II (MPS II): resumo dos achados dos estudos incluídos na presente revisão sistemática.

\begin{tabular}{|c|c|c|c|c|c|}
\hline Desfechos & Muenzer et al. 13 & Muenzer et al. 14 & Okuyama et al. 15 & Muenzer et al. 16 & Brands et al. 17 \\
\hline Alterações cardíacas & NA & NS & NA & NS & $\begin{array}{c}\downarrow \text { septo } \\
\text { interventricular } \\
\text { diástole } \\
\downarrow \text { índice massa VE }\end{array}$ \\
\hline $\begin{array}{l}\text { Capacidade vital forçada (\% } \\
\text { previsto) }\end{array}$ & NS & NS & NS & NS & NA \\
\hline $\begin{array}{l}\text { Capacidade funcional } \\
\text { (CHAQ-DIS) }\end{array}$ & NA & $?$ & NA & NA & NA \\
\hline Esplenomegalia & $\downarrow$ & $\downarrow$ & $\downarrow$ & $\downarrow$ & NA \\
\hline Hepatomegalia & $\downarrow$ & $\downarrow$ & $\downarrow$ & $\downarrow$ & NA \\
\hline GAGs urinários & $\downarrow$ & NA & NA & NA & NA \\
\hline $\begin{array}{l}\text { Teste da caminhada de } 6 \\
\text { minutos (distância percorrida) }\end{array}$ & $\uparrow$ & NS & NS & $\uparrow$ & NA \\
\hline $\begin{array}{l}\text { Síndrome da apneia obstrutiva } \\
\text { do sono (Índice apneia- } \\
\text { hipopneia) }\end{array}$ & NA & $\downarrow$ & $\uparrow$ & NA & NA \\
\hline Velocidade de crescimento & NA & NA & NA & $?$ & NA \\
\hline
\end{tabular}

CHAQ-DIS: Child Health Assessment Questionnaire Disability Index Score; GAGs: glicosaminoglicanos; NA: não avaliado; NS: não significativo; $\uparrow$ : aumento; $\downarrow$ : redução; ?: inconclusivo; VE: ventrículo esquerdo.

Nota: Os desfechos definidos a priori como relevantes pela equipe do presente estudo, mas que não foram avaliados pelos estudos incluídos na revisão sistemática, foram: acuidade visual, custo-efetividade, distúrbio de comportamento, estado nutricional, estágio puberal, estatura, hidrocefalia, mielopatia, número de episódios de otites, número de infecções respiratórias, número de internações hospitalares, óbito, opacificação de córnea, peso, qualidade de vida, regressão neurológica, resistência física, retardo mental, sobrevida.

na amplitude articular do cotovelo ( $\mathrm{p}=0,0476$ - sem demonstração da modificação em graus) na comparação da IDS semanal com placebo 13 . O outro ECR não reporta os resultados desse desfecho, justificando terem ocorrido dificuldades na interpretação, em virtude de heterogeneidade dos pacientes e das aferições 14 .

\section{f) Excreção urinária de GAGs}

A comparação com placebo é demonstrada em um dos ECRs: houve redução na excreção de GAGs urinários após 53 semanas, na comparação de ambos os regimes de IDS com placebo ( $\mathrm{p}<$ 0,0001 para cada grupo), sendo maior no grupo que recebeu TRE semanalmente do que no que passou pela terapia quinzenalmente $(\mathrm{p}=0,039)$. Ao final de 53 semanas de tratamento, 26/64 $(40,6 \%)$ dos pacientes atingiram valores normais deste marcador bioquímico 13 .
- Comparação 2: síntese dos efeitos da idursulfase em intervenções sem grupo de controle

\section{a) TC $6 \mathrm{~min}$}

O estudo de Muenzer et al. 14 apresenta os resultados sem estabelecer comparação com placebo, somando a fase duplo-cega e aberta: após 12 meses houve modificação nos grupos $0,5 \mathrm{mg} / \mathrm{kg} \mathrm{e}$ $1,5 \mathrm{mg} / \mathrm{kg}$ (média $\pm \mathrm{DP}=10,9 \pm 7,15 \%$ e $27,9 \pm 15,1 \%$ de variação na distância caminhada em metros, respectivamente), contudo não há avaliação estatística entre os valores finais e inicias para cada grupo ou da diferença entre eles. Dentre os estudos abertos, um não encontrou diferenças entre pré- e pós-TRE $(\mathrm{p}=0,109) 15$, já o outro apontou aumento estatisticamente significativo após 36 meses $(\mathrm{p}<0,01)$, sem relatar valores absolutos ao final do período 16 .

A metanálise pôde ser realizada utilizandose dois estudos 15,16. Houve incremento médio de 43,5m (IC95\%: 25,1-61,9) na distância percor- 
rida no TC 6 min (Figura 2a). A heterogeneidade estatística não foi significativa (teste Q de Cochran, $\mathrm{p}=0,664$ e I2 $=0 \%$ ), embora o número de estudos seja muito limitado para esse tipo de inferência 20 .

\section{b) Função pulmonar}

Este desfecho foi aferido em quatro estudos, sendo a diferença estatisticamente significativa apenas na CVF absoluta em um dos estudos 13,14,15,16. Muenzer et al. 14 mostram resultados somando a fase duplo-cega e aberta: em relação ao início da TRE, não houve diferença no Volume Expiratório forçado em um segundo (VEF1) $(p=0,61)$ ou CVF\% $(p=0,08) 14$, resultado semelhante para CVF\% em outro estudo aberto $(p=0,025) 15$. Muenzer et al. 16 demonstraram aumento na CVF\% aos 16 meses de estudo, mas não ao término deste (p não demonstrado).

A metanálise pôde ser realizada utilizando-se dois estudos 15,16. Houve modificação média de 0,229L (IC95\%: 0,028-0,429) na CVF absoluta e de 10,8\% (IC95\%: 6,3-15,3) na CVF\% (Figura 2b) $\left[\mathrm{p}(\mathrm{Q})=0,036 ; \mathrm{I}^{2}=77,2 \%\right.$ e $\mathrm{p}(\mathrm{Q})=0,00 ; \mathrm{I}^{2}=94,7 \%$, respectivamente].

\section{c) Volume hepático e esplênico}

Foi verificada redução estatisticamente significativa da hepatoesplenomegalia após 6 e 12 meses de tratamento ( $p<0,0001$ ), nos resultados combinados das fases aberta e controlada por placebo em Muenzer et al. 13. Esse resultado também foi encontrado em Okuyama et al. 15 (redução média $\pm \mathrm{EP}$ do volume hepático: $-498,0 \pm 70,2 \mathrm{~mL}$; $\mathrm{p}=0,002$ e do esplênico: $-72,1 \pm 15,7 ; \mathrm{p}=0,002$ ) e em Muenzer et al. 16, cuja magnitude média da redução está demonstrada apenas graficamente e se situa ao redor de $240 \mathrm{~mL}$ para o fígado e $60 \mathrm{~mL}$ para o baço 16 ( $\mathrm{p}<0,001$ para ambos).

\section{d) Alterações cardíacas}

As alterações cardíacas foram avaliadas por ecocardiografia em três estudos 14,16,17. Houve redução significativa da dimensão do septo interventricular durante a diástole em um estudo $(-0,36$ escore $\mathrm{Z} /$ ano, $\mathrm{p}=0,05 ; \mathrm{n}=5)$ e do índice de massa ventricular esquerda $(-0,26$ escore $\mathrm{Z}$ /ano, $\mathrm{p}=0,032 ; \mathrm{n}=5$ ) 17 , sem modificações significativas na fração de ejeção, morfologia valvar ou outros parâmetros cardíacos, após tratamento com IDS 14,16,17.

\section{e) Mobilidade articular}

Nos estudos sem comparação com placebo, houve ganho estatisticamente significativo na flexão de quadril (média \pm EP 14,2 \pm 5,1o; teste de Wilcoxon para mudança percentual na comparação pré- e pós-tratamento $\mathrm{p}=0,031$ ) 15 e na amplitude articular do ombro (abdução, flexão-extensão, ao redor de 12o; teste de Wilcoxon $\mathrm{p}<0,005$ ), demonstrado apenas graficamente 16 .

\section{f) Apneia obstrutiva do sono}

A modificação da apneia obstrutiva do sono foi avaliada em dois estudos. Um dos ECRs relata os resultados pré- e pós-tratamento com IDS, sem compará-lo ao placebo ou apresentar análises estatísticas: houve estabilidade nos índices de apneia-hipopneia e redução média de 17 eventos/hora de dessaturação de oxigênio 14. Já um estudo aberto demonstrou aumento no índice de

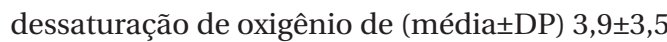
eventos/hora $(n=8)$, sobretudo devido à piora de um único paciente e estabilidade dos demais 15.

\section{g) Capacidade funcional}

O estado funcional e a capacidade para realizar atividades físicas diárias foram avaliados em estudo não controlado, mediante o questionário Child Health Assessment Questionnaire Disability Index Score (CHAQ-DIS) 16. O instrumento foi aplicado aos pacientes maiores de 12 anos e a todos os pais ou responsáveis; houve modificações clinicamente significativas nos meses intermediários para ambos os grupos, porém os valores apontados referem-se ao 24o mês ( $\mathrm{p}=$ 0,047 e p $=0,031$, pais e crianças respectivamente), sem que haja relato referente aos pais ao término do estudo.

\section{h) Estatura e velocidade de crescimento}

O ganho estatural dos pacientes foi reportado em um dos estudos, sem análise estatística ou comparação com pacientes não tratados 16. Os resultados foram apresentados para dois grupos: (1) pacientes que iniciaram a TRE com menos de dez anos de idade $(n=9)$ que demonstraram crescimento médio (DP) de $14,6 \pm 5,5 \mathrm{~cm}$, permanecendo com peso e altura na curva de normalidade de acordo com o Centers for Disease Control and Prevention (CDC; Estados Unidos) 21; (2) pacientes que iniciaram TRE com mais de dez anos de idade $(n=9)$ apresentavam baixa estatura no início da TRE (mediana $130 \mathrm{~cm}$ ) e cresceram em média $8,1 \pm 1,6 \mathrm{~cm}$ após três anos. 
Figura 2

Gráfico de metanálise (forest plot).

2a) Efeito da idursulfase na distância percorrida durante o teste da caminhada de seis minutos.

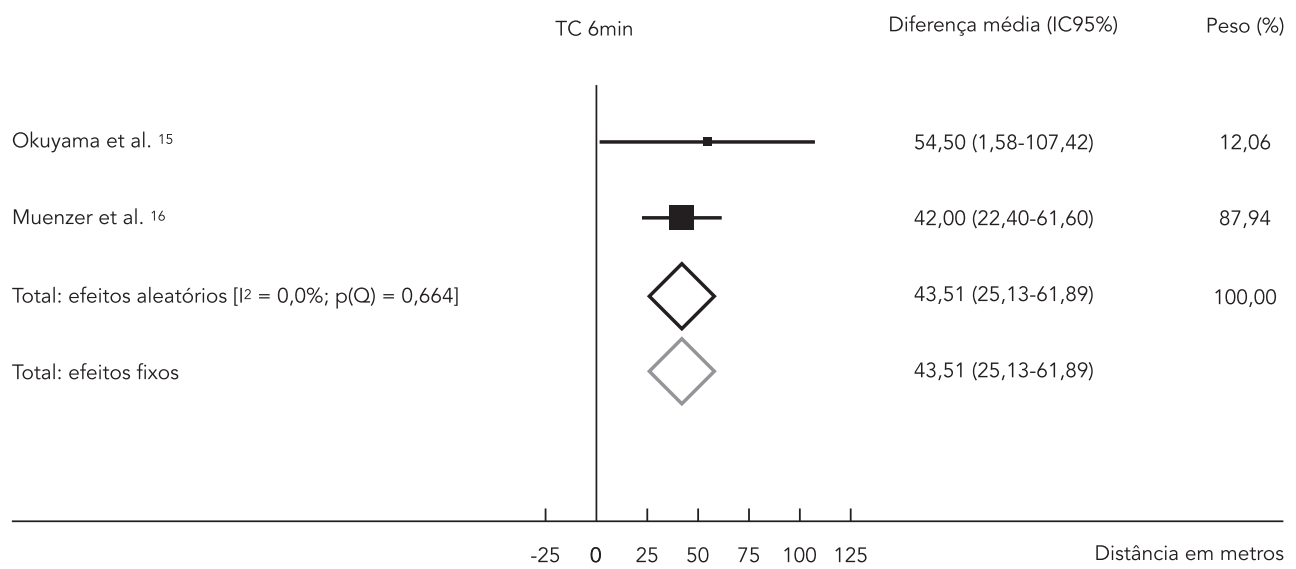

2b) Efeito da idursulfase na capacidade vital forçada (em \% do previsto).

Okuyama et al. 15
Total: efeitos aleatórios $[12=94,7 \% ; p(Q)<0,001]$
Total: efeitos fixos

TC 6min: teste da caminhada de seis minutos.

\section{i) Excreção urinária de GAGs}

Houve redução significativa deste marcador bioquímico, seja na comparação ao placebo 13,14 ou aos valores pré-TRE 15,16. É interessante observar que muitos pacientes atingem valores normais após alguns meses de tratamento 14, e tais mudanças parecem manter-se num platô a partir de então 16 .

\section{Segurança}

\section{- Eventos adversos}

Os eventos adversos graves, com risco de vida que ocorreram durante a TRE, estiveram dentro do esperado para a história natural da MPS II e não foram relacionados ao medicamento em estudo 13,14,15,16.

Os eventos adversos relacionados à infusão mais comuns foram: cefaleia, febre, reações cutâneas (urticária e eritema) 13,14,16, dispneia, dor abdominal e síncope vasovagal 15 . Os con- 
siderados graves incluíram estresse respiratório 14 , tosse, urticária 16 e síncope vasovagal 15 . Os autores relatam que os eventos adversos foram amenizados com aumento do tempo de infusão e pré-medicação com anti-histamínicos e/ou corticosteroides 13,14,15,16.

Foi possível realizar metanálise de dois estudos que avaliaram os eventos adversos relacionados à infusão de IDS 13,15: 63\% dos pacientes apresentaram ao menos um evento adverso relacionado à infusão. Não houve heterogeneidade estatística significativa $\left[\mathrm{p}(\mathrm{Q})=0,28, \mathrm{I}^{2}=12,6 \%\right]$.

\section{Anticorpos}

Houve desenvolvimento de anticorpos IgG antiidursulfase em 50\%-60\% dos pacientes 14,16 . Foi possível realizar metanálise de dois estudos 13,15: $52 \%$ dos pacientes desenvolveram IgG anti-IDS. Uma ressalva é a grande heterogeneidade entre os estudos para esta variável $\left[\mathrm{p}(\mathrm{Q})=0,015, \mathrm{I}^{2}=\right.$ $76,3 \%]$.

\section{Discussão}

A proposta da presente revisão sistemática com metanálise foi a de avaliar criticamente a literatura disponível sobre TRE na MPS II, buscando estabelecer a eficácia e segurança dessa intervenção. Os estudos investigados incluíram pequeno número de pacientes, com manifestações fenotípicas variáveis e nem sempre correspondentes à realidade clínica, como, por exemplo, exclusão de pacientes com doença neurológica. Apesar das limitações, buscou-se avaliar criticamente a melhor evidência disponível. Dentre os estudos selecionados, houve grande disparidade nos desfechos utilizados pelos autores, os quais incluíram predominantemente desfechos substitutos e relato heterogêneo e limitado de resultados, o que prejudicou a comparação entre os diferentes trabalhos e, portanto, a obtenção de dados suficientes para realizar metanálise com todas as variáveis. Os artigos incluídos relataram apenas dez entre os trinta desfechos definidos a priori pela equipe como relevantes, além de não avaliarem desfechos definitivos (hard endpoints), como a mortalidade.

A única revisão sistemática existente sobre o tema, publicada em 2011, utilizou como critério de inclusão apenas ECRs e quase-experimentos avaliando a TRE versus placebo, ou qualquer outra opção de tratamento 18. Dessa forma, apenas o estudo de Muenzer et al. 13, publicado em 2006, foi incluído e discutido, levando os autores à conclusão de que a TRE com IDS é eficaz em relação à capacidade funcional (TC 6min e CVF), volume hepático e esplênico e na excreção de GAGs urinários dos pacientes com MPS II. Todavia, os autores concluíram que as evidência são limitadas e mais estudos são necessários sobre a eficácia e segurança desse tratamento a longo prazo 18.

O desfecho substituto, distância percorrida no TC 6min, é utilizado para avaliação do sistema cardiopulmonar, já que integra a capacidade respiratória, cardiovascular e o sistema musculoesquelético. Para realizá-lo satisfatoriamente, é necessário que o indivíduo compreenda a tarefa e seja colaborativo, o que pode dificultar o teste em crianças e pacientes com alterações neurocognitivas. Consideram-se como mudanças clinicamente significativas aquelas maiores que modificações por erros de aferição ou por variabilidade diária. Um dos parâmetros mais utilizados na literatura, o da American Thoracic Society (ATS), considera o ponto de corte de $54 \mathrm{~m}$ (IC95\%: 37-71) como clinicamente significativo ${ }^{22}$. A metanálise realizada neste artigo, ao comparar os efeitos pré- e pós-tratamento, demonstrou que a IDS foi capaz de aumentar a distância percorrida no TC 6min em 43,5m (IC95\%: 25,1-61,9), porém as modificações médias não foram clinicamente relevantes.

A eficácia da IDS na função pulmonar foi avaliada por intermédio da CVF, aferida por espirometria. Consideram-se clinicamente relevantes aumentos superiores a $11 \%-15 \%$ (> 200mL) no VEF1 e na CVF em relação aos valores prévios ao tratamento, pois $8 \%$ (ou $150 \mathrm{~mL}$ ) podem ser atribuídos apenas à própria variabilidade da realização da espirometria 23,24. Também é interessante observar que a capacidade pulmonar possui relação linear com a estatura do indivíduo, podendo este ser um fator de confusão em populações em fase de crescimento. Conforme os estudos avaliados, a metanálise da comparação pré- e pós-tratamento demonstrou que a IDS foi capaz de aumentar a CVF absoluta em 0,229L (IC95\%: 0,028-0,429) e a CVF\% prevista em 10,8\% (IC95\%: 6,3-15,3) nos pacientes com MPS II de diversas faixas etárias. No entanto, tal benefício não é considerado de repercussão clínica significativa nos parâmetros de capacidade pulmonar.

Houve redução estatisticamente significativa da hepatoesplenomegalia em todos os estudos que avaliaram este desfecho 13,14,15,16. É interessante observar que adultos e crianças foram avaliados e que os resultados são demonstrados de forma incompleta; além disso, a magnitude absoluta dessa redução pode representar diferenças para estas faixas etárias, uma vez que as superfícies corporais diferem.

Dentre os estudos avaliados, houve modificação em parâmetros cardíacos (dimensão do septo interventricular durante a diástole e do 
índice de massa ventricular esquerda) em apenas um estudo 15. Da mesma forma, na modificação da apneia obstrutiva do sono e do índice de dessaturação de oxigênio, o papel da TRE foi inconclusivo 14,15 .

A mobilidade articular não foi avaliada satisfatoriamente após a TRE, seja por dificuldades metodológicas de aferição, seja pela heterogeneidade clínica, seja pela amplitude etária dos pacientes envolvidos. Não encontramos na literatura quais seriam os valores considerados clinicamente relevantes, mas alguns autores, avaliando MPS VI, consideram arbitrariamente o aumento superior a 10o como significativo 25,26. Portanto, houve mudanças clinicamente relevantes na mobilidade articular do cotovelo (diferença média não relatada) 13 , ombro (aproximadamente 12o, abdução, flexão-extensão) 16 e quadril (aproximadamente 14ㅇ, flexão), este último avaliado apenas por um pequeno estudo ( $\mathrm{n}=10$ pacientes) ${ }^{15}$. É possível que a TRE promova aumento da amplitude do movimento articular, entretanto a paucidade de resultados não nos permite conclusões sólidas a esse respeito.

Apenas um estudo, aberto e sem grupo-controle, avaliou o estado funcional dos pacientes e sua capacidade para realizar atividades físicas diárias. O questionário CHAQ-DIS foi aplicado aos pacientes e a seus pais, apontando modificações clinicamente significativas 16 , porém, na ausência de um grupo-controle, não é possível inferir se a TRE foi responsável por essa mudança positiva ou se outros fatores não mensurados estão envolvidos.

A avaliação do ganho estatural da coorte de pacientes originalmente incluídos no ECR fase II/III 13 foi relatada em um estudo de seguimento 16 . Este demonstrou que a velocidade linear de crescimento nos pacientes pré-púberes esteve ao redor de $4,33 \mathrm{~cm} / \mathrm{ano}$, o que seria próximo ao esperado para crianças sem MPS II (cerca de 5-6cm/ano) 21; o estudo indicou ainda que os pacientes mantiveram sua estatura distante até -1DP da média para sua idade. Todavia, a ausência de um grupo para comparação dificulta a conclusão sobre as modificações da história natural da doença mediante o uso da TRE.

A excreção de GAGs urinários (taxa GAG/creatinina urinária) foi inicialmente estabelecida como uma ferramenta de rastreio e diagnóstico para os diferentes tipos de MPS 27,28. Não há evidência de que este marcador bioquímico seja útil para monitorar a carga corporal total, uma vez que provavelmente represente o depósito renal de GAGs 29. Em adição, ele varia de acordo com a estatura, peso e idade, podendo ser afetado por dano tecidual e função renal 29, variando amplamente para cada indivíduo e dificultando sua interpretação. Contudo, na ausência de outros marcadores úteis, ele geralmente é estudado, já que a urina é um material de fácil coleta e análise. Houve redução de valores estatisticamente significativa, em comparação ao placebo ou aos valores pré-TRE 13,14,16. É interessante observar que muitos pacientes atingem os valores normais após alguns meses de tratamento 13 , mas tais mudanças parecem manter-se num platô a partir de então 16, sugerindo que as manifestações da doença podem estabilizar-se com o tempo ou, o que parece mais provável, a excreção urinária de GAGs não teria utilidade como ferramenta para monitorização da TRE a longo prazo 30.

A exemplo dos GAGs urinários, questiona-se se outros parâmetros não estabilizariam após um determinado período de TRE. Os estudos publicados até o momento, porém, não são capazes de responder a tais perguntas por diferentes motivos: ausência de grupo-controle, curto tempo de seguimento, heterogeneidade clínica dos pacientes ou pequena sobreposição de desfechos avaliados.

De modo geral, a IDS foi considerada segura, sendo frequentes os eventos adversos relacionados à infusão, mas passíveis de serem controlados com aumento do tempo de pré-tratamento com anti-histamínicos e/ou corticosteroides. O desenvolvimento de anticorpos IgG anti-idursulfase durante a TRE também foi comum, mas os efeitos destes anticorpos sobre a eficácia do medicamento ainda não estão elucidados.

É válido mencionar um estudo de coorte patrocinado pelo NIHR Health Technology Assessment Programme, programa de investigação nacional para o serviço nacional de saúde do Reino Unido (NHS). O artigo, que analisou o custo-efetividade da TRE em MPS II ( $\mathrm{n}=58)$ e em outras doenças lisossômicas, não foi incluído na presente revisão por reunir dados retrospectivos além dos prospectivos. A única constatação estatisticamente significativa entre os pacientes com MPS II do trabalho foi a relação entre a duração da TRE e o crescimento em crianças 31 .

A alternativa existente à TRE seria o TCTH, porém esta terapia tem limitações importantes associadas à alta morbimortalidade, como desenvolvimento de doença do enxerto versus hospedeiro, rejeição do enxerto e infecções após o procedimento ${ }^{31}$. A principal vantagem dessa estratégia em relação à TRE seria o potencial efeito sobre a doença neurológica, haja vista que a IDS provavelmente não ultrapassa a barreira hematoencefálica; todavia, na literatura, são escassos os relatos prospectivos sobre TCTH na MPS II. O maior deles avaliou desfechos relevantes e incluiu nove pacientes com idade entre 3 e 16 anos ${ }^{32}$. No período de observação (7 a 17 anos), 
as anormalidades cardiovasculares se estabilizaram, a hepatoesplenomegalia se resolveu, os defeitos de transmissão auditiva e a rigidez articular melhoraram e os desfechos neuropsicológicos foram variáveis. Assim, o TCTH parece ter alguns resultados favoráveis, mas ainda não está claro o efeito do tratamento na progressão de desfechos neurológicos 32 .

\section{Conclusões}

As evidências disponíveis na literatura sobre a TRE com IDS no tratamento da MPS II são escassas, sendo necessários estudos adicionais para que se estabeleça a real capacidade da TRE em modificar a história natural da MPS II, a relevância clínica de tais mudanças, bem como a identificação de possíveis subgrupos que poderiam se favorecer da terapia. Com base nos dados aqui apresentados, verifica-se que a TRE com IDS pode trazer alguns benefícios aos indivíduos com MPS II, pois a sua administração associou-se não só à redução da excreção de GAGs urinários e da hepatoesplenomegalia em todos os estudos avaliados, como também ao aumento na mobilidade de quadril e ombro em uma pequena série de casos. Além disso, houve aumento da distância percorrida no TC $6 \mathrm{~min}$ e da CVF\%, porém sem impacto clinicamente relevante. O desenvolvimento de outras estratégias de tratamento para MPS II, incluindo formas alternativas de aplicação da IDS, deve ser incentivado.

\section{Implicações para a prática}

Como a MPS II é uma doença rara, o número de pacientes disponíveis para realização de ensaios clínicos é limitado, o que diminui o poder estatístico para análise de desfechos relevantes. O princípio que norteia o tratamento das doenças de incidência extremamente baixa esbarra em questões bioéticas, como equidade e escassez de recursos. Este amplo conjunto de desafios significa que as doenças raras e os agentes utilizados no seu tratamento exigem formas específicas de avaliação de eficácia e segurança. Apesar dessa dificuldade, há necessidade de estudos com melhor qualidade metodológica para avaliação da TRE com IDS. Os dados atualmente disponíveis sobre a segurança e a eficácia desse medicamento tendem a justificar a sua prescrição, em virtude, principalmente, da ausência de alternativas comprovadamente eficazes para tratamento.

\section{Resumen}

La mucopolisacaridosis tipo II (MPS II) es una enfermedad genética de amplio espectro clínico, caracterizada por una deficiencia de la enzima iduronato-2-sulfatasa. El objetivo fue evaluar la seguridad y eficacia de la Terapia de Reemplazo Enzimático (TRE) con idursulfasa (IDS) en la MPS II. En las bases PubMed/MEDLINE, EMBASE, LILACS y Cochrane Library se inició la búsqueda hasta el 30 de noviembre de 2012. Sólo cinco estudios cumplieron los criterios de inclusión (ensayos controlados aleatorios -ECA, o ECA abiertos o series de casos prospectivo incluyendo $\geq 5$ pacientes y evaluación de los resultados pertinentes). El metaanálisis se realizó para la capacidad vital forzada (FVC; absoluta y \%) y la distancia caminada en 6 minutos, con cambios significativos en ambas variables; el riesgo también se encuentra aumentado por reacciones leves y anticuerpos IgG, relacionados con la infusión con IDS. El TRE con IDS es seguro y tiene un beneficio potencial en la MPS II, pero se necesitan estudios adicionales.

Mucopolisacaridosis; Terapia de Reemplazo Enzimático; Evaluación de Tecnologías de Salud 


\section{Colaboradores}

T. Alegra participou da elaboração das estratégias da revisão sistemática, buscas nas bases de dados, seleção dos artigos, extração dos dados e elaboração do artigo. D. P. Eizerik participou da elaboração das estratégias da revisão sistemática, extração dos dados, análise dos resultados e revisão do artigo. C. C. S. Cerqueira participou das buscas nas bases de dados, seleção dos artigos e revisão do artigo. T. V. Pereira participou da avaliação dos dados, organização dos resultados, análise estatística, realização de metanálise, redação e revisão do manuscrito. A. D. Dornelles participou da revisão do processo de busca e seleção, organização do fluxograma e revisão do artigo. I. V. D. Schwartz participou da elaboração das estratégias da revisão sistemática, análise e revisão dos resultados e elaboração do artigo.

\section{Agradecimentos}

Aos Drs. Luiz Carlos Santana, Carolina F. Moura de Souza e Filippo Vairo, os quais colaboraram para a realização deste estudo. A pesquisa foi financiada conjuntamente pelo Ministério da Ciência e Tecnologia, Ministério da Saúde e CNPq (MS-SCTIE-DECIT37/2008). Os financiadores não desempenharam qualquer papel no projeto de estudo, coleta de dados e análise, decisão de publicar ou preparação do manuscrito.

\section{Conflito de interesses}

Não declarado.

\section{Referências}

1. Neufeld EF, Muenzer J. The mucopolysaccharidoses. In: Scriver CR, editor. The metabolic and molecular bases of inherited disease. New York: McGraw-Hill; 2001. p. 3421-52.

2. Schwartz IV, Ribeiro MG, Mota JG, Toralles MB, Correia P, Horovitz D, et al. A clinical study of 77 patients with mucopolysaccharidosis type II. Acta Paediatr Suppl 2007; 96:63-70.

3. Scarpa M. Mucopolysaccharidosis type II. In: Pagon RA, Bird TD, Dolan CR, Stephens K, editors. GeneReviews. Seattle: University of Washington, Seattle; 1993-2007. http://www.ncbi.nlm.nih.gov/ books/NBK1116/.

4. Wraith JE. Enzyme replacement therapy with idursulfase in patients with mucopolysaccharidosis type II. Acta Paediatr Suppl 2008; 97:76-8.

5. Schwartz IVD, Pinto LLC, Breda G, Lima L, Ribeiro MG, Mota JG, et al. Clinical and biochemical studies in mucopolysaccharidosis type II carriers. J Inherit Metab Dis 2009; 32:732-8.

6. de Camargo Pinto LL, Maluf SW, Leistner-Segal S, Zimmer da Silva C, Brusius-Facchin A, Burin MG, et al. Are MPS II heterozygotes actually asymptomatic? A study based on clinical and biochemical data, X-inactivation analysis and imaging evaluations. Am J Med Genet A 2001; 155A:50-7.
7. Schwartz IV, Souza CFM, Giugliani R. Tratamento de erros inatos do metabolismo. J Pediatr (Rio J) 2008; 84(4 Suppl):S8-19.

8. Higgins JPT, Green S (editors). Cochrane handbook for systematic reviews of interventions version 5.0.2 [updated September 2009]. The Cochrane Collaboration; 2011.

9. Guyatt G, Vist G, Falck-Ytter Y, Kunz R, Magrini $\mathrm{N}$, Schunemann H. An emerging consensus on grading recommendations? Evid Based Med 2006; 11:2-4.

10. Guyatt GH, Oxman AD, Vist GE, Kunz R, Falck-Ytter Y, Alonso-Coello P, et al. GRADE: an emerging consensus on rating quality of evidence and strength of recommendations. BMJ 2008; 336:924-6.

11. Guyatt GH, Oxman AD, Schünemann HJ, Tugwell P, Knottnerus A. GRADE guidelines: a new series of articles in the Journal of Clinical Epidemiology. J Clin Epidemiol 2011; 64:380-2.

12. Balshem $H$, Helfand M, Schünemann HJ, Oxman AD, Kunz R, Brozek J, et al. GRADE guidelines: 3 . Rating the quality of evidence. J Clin Epidemiol 2011; 64:401-6. 
13. Muenzer J, Wraith JE, Beck M, Giugliani R, Harmatz P, Eng CM, et al. A phase II/III clinical study of enzyme replacement therapy with idursulfase in mucopolysaccharidosis II (Hunter syndrome). Genet Med 2006; 8:465-73.

14. Muenzer J, Gucsavas-Calikoglu M, McCandless SE, Schuetz TJ, Kimura A. A phase I/II clinical trial of enzyme replacement therapy in mucopolysaccharidosis II (Hunter syndrome). Mol Genet Metab 2007; 90:329-37.

15. Okuyama T, Tanaka A, Suzuki Y, Ida H, Tanaka T, Cox GF, et al. Japan Elaprase Treatment (JET) study: idursulfase enzyme replacement therapy in adult patients with attenuated Hunter syndrome (Mucopolysaccharidosis II, MPS II). Mol Genet Metab 2010; 99:18-25.

16. Muenzer J, Beck M, Eng CM, Giugliani R, Harmatz P, Martin R, et al. Long-term, open-labeled extension study of idursulfase in the treatment of Hunter syndrome. Genet Med 2011; 13:95-101.

17. Brands MM, Frohn-Mulder IM, Hagemans ML Hop WC, Oussoren E, Helbing WA, et al. Mucopolysaccharidosis: cardiologic features and effects of enzyme-replacement therapy in 24 children with MPS I, II and VI. J Inherit Metab Dis 2013; 36;227-34.

18. Da Silva EM, Strufaldi MW, Andriolo RB, Silva LA. Enzyme replacement therapy with idursulfase for mucopolysaccharidosis type II (Hunter syndrome). Cochrane Database Syst Rev 2011; (11):CD008185.

19. O'Brien PC. Procedures for comparing samples with multiple endpoints. Biometrics 1984; 40:1079-87.

20. Pereira TV, Patsopoulos NA, Salanti G, Ioannidis J. Critical interpretation of Cochran's $Q$ test depends on power and prior assumptions about heterogeneity. Res Synth Methods 2010; 1:149-61.

21. Kuczmarski RJ, Ogden CL, Guo SS, GrummerStrawn LM, Flegal KM, Mei Z, et al. 2000 CDC growth charts for the United States: methods and development. Vital Health Stat 11 2002; (246):1190.

22. American Thoracic Society Committee on Proficiency Standards for Clinical Pulmonary Function Laboratories. American Thoracic Society statement: guidelines for the six-minute walk test. Am J Respir Crit Care Med 2002; 166:111-7.

23. American Thoracic Society. Lung function testing: selection of reference values and interpretative strategies. Am Rev Respir Dis 1991; 144:1202-18.

24. Pellegrino R, Viegi G, Brusasco V, Crapo RO, Burgos $\mathrm{F}$, Casaburi R, et al. Interpretative strategies for lung function tests. Eur Respir J 2005; 26:948-68.
25. Harmatz P, Kramer WG, Hopwood JJ, Simon J, Butensky E, Swiedler SJ, et al. Pharmacokinetic profile of recombinant human $\mathrm{N}$-acetylgalactosamine 4-sulphatase enzyme replacement therapy in patients with mucopolysaccharidosis VI (MaroteauxLamy syndrome): a phase I/II study. Acta Paediatr Suppl 2005; 94:61-8.

26. Harmatz P, Giugliani R, Schwartz I, Guffon N, Teles EL, Miranda MC, et al. Enzyme replacement therapy for mucopolysaccharidosis VI: a phase 3 , randomized, double-blind, placebo-controlled, multinational study of recombinant human $\mathrm{N}$-acetylgalactosamine 4-sulfatase (recombinant human arylsulfatase B or rhASB) and follow-on, open-label extension study. J Pediatr 2006; 148:533-9.

27. Byers S, Rozaklis T, Brumfield KL, Ranieri E, Hopwood J. Glycosaminoglycan accumulation and excretion in the mucopolysaccharidosis: characterization and basis of diagnostic test for MPS. Mol Genet Metab 1998; 65:285-90.

28. Gallegos-Arreola MP, Machorro-Lazo MV, FloresMartínez SE, Zúñiga-González GM, Figuera LE, González-Noriega A, et al. Urinary glycosaminoglycan excretion in healthy subjects and in patients with mucopolysaccharidoses. Arch Med Res 2000; 31:505-10.

29. Mabe P, Valiente A, Soto V, Cornejo V, Raimann E. Evalulation of reliability of urine mucopolysaccaridosis screening by dimethylmethylene blue and berry spot tests. Clin Chim Acta 2004; 345:135-40.

30. Glamuzina E, Fettes E, Bainbridge K, Crook V, Finnegan N, Abulhoul L, et al. Treatment of mucopolysaccharidosis type II (Hunter syndrome) with idursulfase: the relevance of clinical trial end points. J Inherit Metab Dis 2011; 34:749-54.

31. Wyatt K, Henley W, Anderson L, Anderson R, Nikolaou V, Stein K, et al. The effectiveness and cost-effectiveness of enzyme and substrate replacement therapies: a longitudinal cohort study of people with lysosomal storage disorders. Health Technol Assess 2012; 16:1-543.

32. Guffon N, Bertrand Y, Forest I, Fouilhoux A, Froissart R. Bone marrow transplantation in children with Hunter syndrome: outcome after 7 to 17 years. J Pediatr 2009; 154:733-7.

Recebido em 31/Jan/2013

Versão final reapresentada em 14/Jul/2013

Aprovado em 19/Jul/2013 